\title{
CMR quantitation of change in mitral regurgitation following transcatheter aortic valve replacement (TAVR): impact on left ventricular reverse remodeling and outcome
}

\author{
Pei G. Chew ${ }^{1}$ - Laura E. Dobson ${ }^{1} \cdot$ Pankaj Garg ${ }^{1} \cdot$ Timothy A. Fairbairn $^{1} \cdot$ Tarique A. Musa $^{1} \cdot$ Akhlaque Uddin $^{1}$. \\ Peter P. Swoboda ${ }^{1}$. James R. Foley ${ }^{1}$. Graham J. Fent ${ }^{1}$ - Louise A. E. Brown ${ }^{1}$. Sebastian Onciul ${ }^{1}$. Sven Plein ${ }^{1}$. \\ Daniel J. Blackman ${ }^{2}$. John P. Greenwood ${ }^{1}$
}

Received: 30 May 2018 / Accepted: 13 August 2018 / Published online: 4 September 2018

(c) The Author(s) 2018

\begin{abstract}
Current echocardiographic data reporting the impact of concomitant mitral regurgitation (MR) on outcome in patients who undergo transcatheter aortic valve replacement (TAVR) are conflicting. Using cardiovascular magnetic resonance (CMR) imaging, this study aimed to assess the impact of MR severity on cardiac reverse remodeling and patient outcome. 85 patients undergoing TAVR with CMR pre- and $6 \mathrm{~m}$ post-TAVR were evaluated. The CMR protocol included cines for left (LV) and right ventricular (RV) volumes, flow assessment, and myocardial scar assessment by late gadolinium enhancement (LGE). Patients were dichotomised according to CMR severity of MR fraction at baseline ('non-significant' vs 'significant') and followed up for a median duration of 3 years. Forty-two (49\%) patients had 'significant MR' at baseline; they had similar LV and RV size and function compared to the 'non-significant MR' group but had greater LV mass at baseline. In those with significant MR at baseline, $77 \%(\mathrm{n}=32)$ had a reduction in MR post-TAVR, moving them into the 'non-significant' category at 6-months, with an overall reduction in MR fraction from 34 to $17 \%(\mathrm{p}<0.001)$. Improvement in MR was not associated with more favourable cardiac reverse remodeling when compared with the 'non-improvers'. Significant MR at baseline was not associated with increased mortality at follow-up. Significant MR is common in patients undergoing TAVR and improves in the majority post-procedure. Improvement in MR was not associated with more favourable LV reverse remodeling and baseline MR severity was not associated with mortality.
\end{abstract}

Keywords Mitral regurgitation · Mitral insufficiency $\cdot$ Transcatheter aortic valve replacement $\cdot$ Cardiovascular magnetic resonance $\cdot$ Late gadolinium enhancement

Abbreviations

AS Aortic stenosis

AVA Aortic valve area

CMR Cardiovascular magnetic resonance

FOV Field of view

LGE Late gadolinium enhancement

Electronic supplementary material The online version of this article (https://doi.org/10.1007/s10554-018-1441-y) contains supplementary material, which is available to authorized users.

John P. Greenwood

j.greenwood@leeds.ac.uk

1 Multidisciplinary Cardiovascular Research Centre (MCRC) \& Leeds Institute of Cardiovascular and Metabolic Medicine (LICAMM), University of Leeds, Leeds LS2 9JT, UK

2 Leeds Teaching Hospitals NHS Trust, Leeds, UK
LV Left ventricular

LVEDP LV end-diastolic pressure

MR Mitral regurgitation

PG Pressure gradient

RV Right ventricular

SD Standard deviation

TAVR Transcatheter aortic valve replacement

VENC Velocity encoded

\section{Introduction}

Transcatheter aortic valve replacement (TAVR) has been shown to reduce mortality and improve patient symptoms and quality of life [1-3], and is an alternative to surgery in intermediate and high-risk patients with severe aortic stenosis (AS) [4]. Whilst moderate or severe mitral regurgitation 
(MR) is seen in up to $48 \%$ of patients undergoing TAVR, it is often left untreated [5-7]. Current literature reporting the impact of concomitant MR on outcome in patients who undergo TAVR are conflicting and are mainly based on echocardiographic data; which can be limited by poor acoustic windows, eccentric jets and geometric assumptions [8]. Cardiovascular magnetic resonance (CMR) imaging is able to quantify MR with high accuracy and reproducibility using a combination of left ventricular (LV) volumetric measurements and aortic flow quantification [9-11]. Tissue characterization is a further unique strength of CMR, offering non-invasive detection of myocardial fibrosis [12]. In a TAVR population however, quantitative serial assessment of MR by CMR has never been specifically studied, despite its objectiveness, reproducibility and accuracy.

The aims of this study were to (1) to quantitate the change in MR severity at 6-months post-TAVR using CMR, (2) identify determinants of improvement in MR and its association with LV reverse remodeling, (3) assess the clinical impact of MR on the outcomes of patients undergoing TAVR.

\section{Methods}

\section{Study design and population}

In this prospective study, 109 patients with severe AS undergoing TAVR between April 2009 and September 2015 at a single tertiary centre (Leeds General Infirmary, Leeds, UK) were evaluated. Severe AS was defined as an echocardiographically derived aortic valve area of $\leq 1.0 \mathrm{~cm}^{2}$, peak aortic velocity of $>4 \mathrm{~m} / \mathrm{s}$ or mean pressure gradient of $>40 \mathrm{mmHg}$. Decision for TAVR intervention was taken by a multidisciplinary heart team in accordance with international guidance. Exclusion criteria included any contraindications to CMR. Baseline clinical, demographic and echocardiographic data were recorded for all patients. CMR scans were performed at baseline and 6-months post-TAVR.

All patients were followed up for a median duration of 3 years and their long-term outcomes were evaluated. Mortality data were obtained from the Office of National Statistics, UK. All patients provided written informed consent. The study was approved by the National Research Ethics Service (08/H1307/106) and complied with the Declaration of Helsinki.

\section{TAVR}

Patients underwent a standard work-up for TAVR which included transoesophageal echocardiography and invasive coronary angiography, with the addition of cardiac computed tomography after 2014. Coronary revascularisation was only performed in those with critical proximal lesions or symptomatic angina. TAVR was performed under general or local anaesthetic using the self-expanding Medtronic CoreValve (Medtronic Inc, Minneapolis, MIN) or the mechanically expanded Boston Lotus valve (Boston Scientific Corporation, Natick, MA) via the femoral or subclavian route by two experienced, high-volume operators. All patients received heparin to maintain an activated clotting time $>250 \mathrm{~s}$ and were treated with dual antiplatelet therapy (aspirin and clopidogrel) for 3-6 months after the procedure.

\section{CMR protocol}

Details of the CMR pulse sequence have been previously described [13]. Briefly, identical baseline preoperative and 6-month postoperative scans were performed on a $1.5 \mathrm{~T}$ MRI system (Intera, Philips Healthcare, Best, Netherlands or Avanto, Siemens Medical Systems, Erlangen, Germany); same scanner vendor used at baseline and 6-months. Multislice, multi-phase cine imaging was performed using a standard steady-state free precession pulse sequence in the short axis (repetition time (TR) $3 \mathrm{~ms}$, echo time (TE) $1.7 \mathrm{~ms}$, flip angle $60^{\circ}$, SENSE factor $2,8 \mathrm{~mm}$ thickness, $0 \mathrm{~mm}$ gap, 30 phases, matrix $192 \times 192$, typical field of view (FOV) $340 \mathrm{~mm}$ ) to cover the entire left and right ventricle. Through-plane velocity encoded (VENC) phase contrast imaging was performed at the aortic sinotubular junction (VENC $250-500 \mathrm{~cm} / \mathrm{s}$, retrospective gating, slice thickness $6 \mathrm{~mm}, 40$ phases, FOV $340 \mathrm{~mm}$ ) or just above the valve prosthesis post-replacement. VENC was typically set at $400-500 \mathrm{~cm} / \mathrm{s}$ on the baseline scan and $250 \mathrm{~cm} / \mathrm{s}$ post-procedure. If aliasing occurred at the pre-set VENC, sequential phase contrast imaging was performed at increasing VENC settings until the aliasing artefact had disappeared.

Late gadolinium enhancement (LGE) imaging (10-12 short axis slices, $10 \mathrm{~mm}$ thickness, matrix $240 \times 240$, typical FOV $340 \mathrm{~mm}$ ) was performed following a Look-Locker sequence (inversion time scout), $10 \mathrm{~min}$ after the administration of $0.2 \mathrm{mmol} / \mathrm{kg}$ of gadoteric acid (Dotarem, Guerbet, Villepinte). Four chamber, two chamber and left ventricular outflow tract (LVOT) views were also obtained as standard. Cross cuts and phase swap imaging were used where necessary for further clarification of the presence/absence of LGE.

\section{CMR analysis}

CMR analysis was performed by two experienced CMR operators (LED, PGC) blinded to clinical details, using dedicated computer software $\left(\mathrm{CVI}^{42}\right.$, Circle Cardiovascular Imaging, Calgary, Alberta, Canada). LV endocardial and epicardial borders were manually contoured (with trabeculation and papillary muscles excluded) at end-diastole and end-systole to allow the calculation of ventricular volumes 
(summation of discs methodology) and LV mass [epicardial volume - endocardial volume multiplied by myocardial density $\left.\left(1.05 \mathrm{~g} / \mathrm{cm}^{3}\right)\right]$; values were indexed to body surface area. Left atrial volume was calculated using the formula:

$\frac{8\left(A 2_{C h}\right)\left(A 4_{C h}\right)}{3 \pi \mathrm{L}}$

where $\mathrm{A} 2_{\mathrm{Ch}}$ and $\mathrm{A} 4_{\mathrm{Ch}}$ refer to the left atrial area in the twochamber and four-chamber views respectively, and $\mathrm{L}$ is the shorter of the two left atrial length measurements. Aortic flow was quantified using cross-sectional phase contrast images with contouring of the aortic lumen to provide aortic forward flow data. MR fraction (\%) was quantified using the equation:

$\frac{\mathrm{LV} \text { stroke volume }- \text { aortic forward flow volume }}{\text { LV stroke volume }} \times 100$

'Significant MR' was defined as MR fraction $>25 \%$ and 'non-significant MR' was defined as MR fraction $\leq 25 \%$ [10]. For the purpose of this study, 'significant MR' represented moderate-severe/severe categories and 'non-significant MR' comprised categories of trivial/mild/moderate as per CMR classification. Changes in the MR severity were assessed between the baseline and 6-month post-procedure scans. Those with a reduction in MR severity grade from 'significant' to 'non-significant' category were classified as 'improvers', and those without (i.e. MR worsened or unchanged) were labelled as 'non-improvers'.

LGE images were reviewed for the presence or absence of hyper-enhancement, which was then classified as either noninfarct pattern (myocardial fibrosis), infarct pattern, or mixed pattern. The number and location of segments containing LGE were classified according to the American Heart Association (AHA) 17-segment model. Myocardial fibrosis was defined as a region of LGE with signal enhancement $>5 \mathrm{SD}$ of the signal intensity of non-enhanced myocardium.

\section{Statistical analysis}

All statistical analysis was performed using the SPSS V.21.0 (IBM Corp., New York, USA). Continuous variables are expressed as mean $\pm \mathrm{SD}$ or median (interquartile range, IQR) in cases of skewed distributions. Categorical variables are expressed as frequencies and percentages. Data were tested for normality using the Shapiro-Wilks test. For normally distributed continuous data, two-tailed unpaired Student's $t$ tests were used for comparisons between groups, and paired Students $t$ tests were used for intra-group comparisons. For non-normally distributed data, Mann-Whitney $U$-test was used. The Chi-Squared test was used for comparing categorical variables.

In order to assess the correlation between dependent and independent variables, Pearson's correlation coefficients were used. Two-sided $\mathrm{P}$ values $<0.05$ were considered statistically significant. Univariate analysis was used to determine predictive factors for MR improvement. Variables with a univariate $p<0.1$ were entered into multi-variable regression analysis. Cumulative survival was analyzed with Kaplan-Meier methodology and log-rank test.

\section{Results}

\section{Patients and baseline characteristics}

From 109 patients with a baseline scan, those with a permanent pacemaker $(n=8)$, severe aortic regurgitation $(n=5)$ or who had an incomplete scan $(n=1)$, were excluded from analysis. Eight patients declined follow up and 2 patients died prior to their 6 month scan. 85 patients with paired CMR scans ( $55 \%$ male gender, mean age $80 \pm 7$ years) who underwent TAVR for severe AS were included in the final data analysis. Basic demographics and clinical data can be seen in Table 1.

In total, 42/85 (49\%) patients were classified as having 'significant MR', and 43/85 (51\%) as 'non-significant MR'. Those with 'significant' MR had a mitral regurgitant volume of $34.5 \pm 9.9 \mathrm{ml}$ and a regurgitant fraction of $34.2 \pm 5.5 \%$. Comparatively, those with 'significant' MR had a greater echocardiographically measured aortic peak forward flow velocity $(4.8 \pm 0.47 \mathrm{~m} / \mathrm{s}$ vs $4.6 \pm 0.51 \mathrm{~m} / \mathrm{s}, \mathrm{p}=0.02)$, although mean pressure gradient and aortic valve area did not differ significantly. The 'significant MR' group $(n=42)$ had similar LV and right ventricular (RV) cavity size and function but had greater LV mass at baseline compared to the 'non-significant MR' group (Table 2). Those with significant MR also had more aortic regurgitation (aortic regurgitant fraction $13.3 \pm 6.3 \%$ vs $9.5 \pm 8.4 \%, p=0.008$ ) by CMR. The presence of LGE was not statistically different between groups ['significant' $21.4 \%(n=9)$ vs 'non-significant' $34.8 \%(\mathrm{n}=15), \mathrm{p}=0.188]$.

\section{Cardiac reverse remodeling following TAVR}

Following TAVR, all patients sustained a significant decrease in their peak aortic valve gradient from $41 \pm 16 \mathrm{mmHg}$ to $18 \pm 10 \mathrm{mmHg}(\mathrm{p}<0.001)$ by CMR. At 6 months, compared to baseline, there were significant reductions in $\mathrm{LV}$ end-diastolic volumes $(\mathrm{p}<0.001), \mathrm{LV}$ end-systolic volumes $(p=0.006)$, and LV mass $(p<0.001)$ (Table 3). Global LV and RV ejection fractions however did not change. In addition, left atrial volumes significantly reduced post-TAVR intervention (Table 3).

The 'significant' MR group had a greater degree of reduction in both MR regurgitant volumes $(-19 \pm 14 \mathrm{ml}$ vs $1 \pm 13 \mathrm{ml}, \mathrm{p}<0.001)$ and MR fraction $(-17 \pm 13 \%$ vs 
Table 1 Baseline demographics in all patients, 'non-significant' and 'significant' MR groups

\begin{tabular}{lllll}
\hline & All patients & $\begin{array}{l}\text { Non-significant MR } \\
(\mathrm{n}=43)\end{array}$ & $\begin{array}{l}\text { Significant MR } \\
(\mathrm{n}=42)\end{array}$ & p value \\
\hline Age at TAVR & $80.2 \pm 4.9$ & $80.1 \pm 7.2$ & $80.2 \pm 7.5$ & 0.93 \\
Male sex, n (\%) & $47(55)$ & $23(53)$ & $24(57)$ & 0.73 \\
Logistic euroscore & $19.8 \pm 13.1$ & $19.6 \pm 13.1$ & $20.0 \pm 13.2$ & 0.80 \\
Euroscore II & $5.45 \pm 4.42$ & $5.4 \pm 4.4$ & $5.5 \pm 4.4$ & 0.80 \\
STS mortality & $4.8 \pm 2.97$ & $5.1 \pm 3.3$ & $4.4 \pm 2.5$ & 0.20 \\
STS morbidity & $23.2 \pm 8.42$ & $23.7 \pm 8.3$ & $22.7 \pm 8.5$ & 0.50 \\
HTN & $44.7 \%$ & $46.5 \%$ & $42.8 \%$ & 0.70 \\
DM & $20.0 \%$ & $20.9 \%$ & $19.0 \%$ & 0.80 \\
AF & $21.2 \%$ & $25.5 \%$ & $16.6 \%$ & 0.30 \\
MI & $22.4 \%$ & $20.9 \%$ & $23.8 \%$ & 0.80 \\
CABG & $29.4 \%$ & $20.9 \%$ & $38.0 \%$ & 0.08 \\
PCI & $25.9 \%$ & $25.5 \%$ & $26.1 \%$ & 0.90 \\
PVD & $21.2 \%$ & $23.2 \%$ & $19.0 \%$ & 0.60 \\
CVA & $15.3 \%$ & $13.9 \%$ & $16.6 \%$ & 0.70 \\
PHT & $37.6 \%$ & $30.2 \%$ & $45.2 \%$ & 0.15 \\
Revascularization pre-TAVR & $8(9)$ & $4(9)$ & $4(10)$ & 0.63 \\
Aortic valve parameters (echocardiogram) & $0.33 \pm 0.84$ & $0.33 \pm 0.09$ & $0.33 \pm 0.07$ & 0.99 \\
AVAi & $4.7 \pm 0.51$ & $4.6 \pm 0.51$ & $4.8 \pm 0.47$ & 0.02 \\
AV max velocity & $49.7 \pm 11.6$ & $47.5 \pm 10.8$ & $51.9 \pm 12.1$ & 0.07 \\
AV mean PG & & & &
\end{tabular}

Data as mean $\pm \mathrm{SD}, \mathrm{n}(\%)$

$A F$ atrial fibrillation, $A V$ aortic valve, $A V A i$ aortic valve area (indexed), $C A B G$ coronary artery bypass graft, $C V A$ cerebrovascular attack, $D M$ diabetes mellitus, $H T N$ hypertension, $M I$ myocardial infarction, $P C I$ percutaneous coronary intervention, $P G$ pressure gradient, $P H T$ pulmonary hypertension, $P V D$ peripheral vascular disease, $S T S$ society of thoracic surgery, TAVR transcatheter aortic valve replacement
$1 \pm 14 \%, \mathrm{p}<0.001)$. No significant change in LV ejection fraction $(0.2 \pm 8 \%$ vs $3 \pm 9 \%, \mathrm{p}=0.15)$, RV ejection fraction $(2 \pm 9 \%$ vs $1 \pm 9 \%, \mathrm{p}=0.54)$ or LV mass $(-32 \pm 19 \mathrm{~g}$ vs $-25 \pm 18 \mathrm{~g}, \mathrm{p}=0.07$ ) were seen between groups. Those with significant MR experienced a greater reduction in LV end-diastolic $(\mathrm{p}<0.001)$ and end-systolic volumes $(\mathrm{p}=0.04)$ when compared to the 'non-significant' MR group (Fig. 1).

\section{Changes in MR fraction in the 'significant MR' group}

In those with significant MR at baseline $(n=42), 77 \%$ $(\mathrm{n}=32)$ had a significant reduction in MR, moving them into the 'non-significant' category at 6 months, with an overall reduction in MR fraction from $34 \pm 6 \%$ to $17 \pm 14 \%$ $(\mathrm{p}<0.001)$ (Fig. 2).

\section{Changes in haemodynamics and cardiac reverse remodeling according to MR 'improver' and 'non-improver' status}

From the total study population, MR significantly improved in $38 \%(n=32)$ of patients 6 -months post-TAVR, and worsened/unchanged in $62 \%(n=53)$ of patients. At follow up, the 'improvers' group, but not the 'non-improvers', had a significant improvement in LV stroke volume index $(\mathrm{p}=0.04)$ and a greater increase in aortic forward flow ( $\mathrm{p}<0.001)$. Improvement in MR however was not associated with more favourable cardiac LV reverse remodeling compared with the 'non-improvers' (Fig. 3).

In the 'improvers' group, $72 \%(n=23)$ had presence of LGE, $22 \%(n=7)$ had no LGE and LGE imaging was not performed in $6 \%(n=2)$ due to severe renal impairment. In those with LGE, the pattern of LGE was non-infarct pattern in $61 \%(n=14)$, infarct pattern in $35 \%(n=8)$, and mixed in $4 \%(\mathrm{n}=1)$. The presence of LGE at baseline was associated with a greater reduction in MR fraction at 6-months following TAVR intervention $(-11 \pm 16 \%$ vs $0.2 \pm 16 \%, \mathrm{p}=0.01)$.

\section{Other factors associated with MR improvement}

Univariate regression analysis was conducted to look for any clinical or CMR factors associated with MR reduction following TAVR. The following variables were tested: baseline demographics, baseline and 6 months- LV and RV ejection fraction, mass, and volumes; pre-treatment and post-treatment mean trans-aortic gradient (see Online Supplementary Appendix). A higher baseline RV ejection fraction or RV stroke volume, and a greater reduction in LV end-diastolic 
Table 2 Baseline CMR characteristics of patients in all patients, 'non-significant' and 'significant' MR groups

\begin{tabular}{|c|c|c|c|c|}
\hline & All patients & $\begin{array}{l}\text { Non-significant } \\
\text { MR }(n=43)\end{array}$ & Significant MR $(n=42)$ & $\mathrm{p}$ value \\
\hline LV mass $(\mathrm{g})$ & $138.2 \pm 35.3$ & $127.5 \pm 31$ & $149 \pm 32.9$ & 0.007 \\
\hline LV mass index $\left(\mathrm{g} / \mathrm{m}^{2}\right)$ & $76.1 \pm 18.3$ & $73.5 \pm 16.5$ & $83.3 \pm 23.3$ & 0.01 \\
\hline LVEDV (ml) & $179 \pm 49.3$ & $170 \pm 44.2$ & $183 \pm 45.3$ & 0.33 \\
\hline LVESV (ml) & $84.2 \pm 43.5$ & $86.7 \pm 50.8$ & $81.7 \pm 34.9$ & 0.59 \\
\hline $\operatorname{LVEF}(\%)$ & $54.8 \pm 12.2$ & $52.5 \pm 13.3$ & $56.3 \pm 11$ & 0.14 \\
\hline $\operatorname{RVEDV}(\mathrm{ml})$ & $139.9 \pm 36.0$ & $135.6 \pm 32.1$ & $144.3 \pm 39.5$ & 0.27 \\
\hline RVEF (\%) & $54.2 \pm 9.5$ & $53.5 \pm 10.7$ & $55.0 \pm 8.8$ & 0.46 \\
\hline LA volume (ml) & $131.8 \pm 45.0$ & $130.9 \pm 51.4$ & $132.8 \pm 38.1$ & 0.85 \\
\hline LA volume index $\left(\mathrm{ml} / \mathrm{m}^{2}\right)$ & $72.8 \pm 24.9$ & $73.0 \pm 28.8$ & $72.6 \pm 20.7$ & 0.94 \\
\hline MR volume (ml) & $22.4 \pm 15.0$ & $10.3 \pm 8.1$ & $34.5 \pm 9.9$ & $<0.001$ \\
\hline MR fraction (\%) & $22.6 \pm 13.3$ & $11.4 \pm 9.0$ & $34.2 \pm 5.5$ & $<0.001$ \\
\hline \multicolumn{5}{|l|}{ Classifications of LGE, $n(\%)$} \\
\hline None & $24(28)$ & $15(35)$ & $9(21)$ & \\
\hline Infarct pattern & $19(22)$ & $10(23)$ & $9(21)$ & \\
\hline Non-infarct pattern & $33(39)$ & $14(33)$ & $19(45)$ & \\
\hline Mixed & $4(5)$ & $2(5)$ & $2(5)$ & \\
\hline Not done & $5(6)$ & $2(5)$ & $3(7)$ & \\
\hline \multicolumn{5}{|l|}{ Presence of LGE n, (\%) } \\
\hline LGE present & $56(66)$ & $15(35)$ & $9(21)$ & 0.188 \\
\hline LGE absent & $24(28)$ & $26(60)$ & $30(71)$ & \\
\hline LGE not done & $5(6)$ & $2(5)$ & $3(7)$ & \\
\hline
\end{tabular}

Data as mean $\pm \mathrm{SD}, \mathrm{n}(\%)$

$L A$ left atrial, $L G E$ late gadolinium enhancement, $L V E D V$ left ventricular end-diastolic volume, $L V E F$ left ventricular ejection fraction, $L V E S V$ left ventricular end-systolic volume, $L V S V$ left ventricular stroke volume, $M R$ mitral regurgitation, $R V E D V$ right ventricular end diastolic volume, $R V E F$ right ventricular ejection fraction pressure (LVEDP) post-TAVR were all significantly associated with MR improvement. A lower aortic forward flow at baseline was also associated with the reduction in MR. Multivariate predictors of improved MR following TAVR intervention were pre-operative absence of atrial fibrillation, a higher RV stroke volume and a lower aortic forward flow at baseline.

\section{Impact of MR on mortality}

At a median of 3 (IQR 2.03-3.97) years follow-up, 24\% $(n=20)$ of TAVR patients had died. MR severity at baseline did not differ between those who died and those who did not; (mortality rate $13 \%$ vs $14 \%$, non-significant vs significant, $\mathrm{p}=0.84$ ) (Fig. 4). Those who died also had a comparable reduction in MR severity post-TAVR $(-7.3 \%$ vs $-8.3 \%$, $\mathrm{p}=0.81$ ). Cumulative survival rates between the 'improvers' and 'non-improvers' did not differ at follow up (mean survival 5.5 years 95\% CI 4.6-6.4 vs 5.5 years 95\% CI 4.7-6.3, improvers vs non-improvers). Residual significant MR was also not associated with increased mortality.

Intra-observer variability for $\mathrm{LV}$ quantitation in this study was $1.6 \%, 3.6 \%, 3.0 \%$ and $1.8 \%$ for LV end-diastolic volume,
LV mass, LV stroke volume and LV ejection fraction respectively; whilst the coefficient of variation for peak aortic flow velocity and aortic forward flow volume was $0.2 \%$ and $1.7 \%$.

\section{Discussion}

This is the first CMR study to specifically assess MR in quantitative terms and evaluate its impact on cardiac reverse remodeling and mortality in patients undergoing TAVR. The main findings were (1) MR was shown to occur frequently in a TAVR population and those with 'significant MR' had a greater LV mass at baseline; (2) The presence of significant MR at baseline did not prevent LV reverse remodeling, as demonstrated by the substantial reduction in LV mass, LV diastolic and systolic volumes; (3) In those with significant MR at baseline, the MR is likely to improve following TAVR without the need for any specific intervention on the mitral valve; (4) The presence of LGE at baseline was associated with a greater improvement in MR at 6-months post-TAVR; (5) Improvement in MR was neither associated with lower mortality nor more favourable cardiac reverse remodeling 
Table 3 CMR parameters pre- and post-TAVR interventions in all patients

\begin{tabular}{|c|c|c|c|}
\hline All patients & $\begin{array}{l}\text { Baseline } \\
\mathrm{n}=85\end{array}$ & $\begin{array}{l}6 \mathrm{~m} \text { follow up } \\
\mathrm{n}=85\end{array}$ & $\mathrm{p}$ value \\
\hline LV mass (g) & $138.2 \pm 35.3$ & $109.9 \pm 31$ & $<0.001$ \\
\hline LVEDV (ml) & $179 \pm 49.3$ & $166.4 \pm 44.2$ & $<0.001$ \\
\hline LVESV (ml) & $84.2 \pm 43.5$ & $75.7 \pm 35.6$ & 0.006 \\
\hline LVSV (ml) & $94.5 \pm 22.5$ & $90.7 \pm 18.7$ & 0.04 \\
\hline $\operatorname{LVEF}(\%)$ & $54.8 \pm 12.2$ & $56.3 \pm 10.6$ & 0.10 \\
\hline RVSV (ml) & $74.3 \pm 18.4$ & $78.7 \pm 20.4$ & 0.04 \\
\hline $\operatorname{RVEF}(\%)$ & $54.2 \pm 9.5$ & $55.4 \pm 10.1$ & 0.20 \\
\hline LA volume $(\mathrm{ml})$ & $131.8 \pm 45.0$ & $119.1 \pm 41.3$ & $<0.001$ \\
\hline MR volume $(\mathrm{ml})$ & $22.4 \pm 15.0$ & $13.7 \pm 12.9$ & $<0.001$ \\
\hline MR fraction (\%) & $22.6 \pm 13.3$ & $14.5 \pm 12.4$ & $<0.001$ \\
\hline \multicolumn{4}{|l|}{ MR classifications (n) } \\
\hline \multicolumn{4}{|l|}{ Mitral regurgitation $\%$} \\
\hline MR none $(0 \%)$ & 8 & 14 & \\
\hline MR mild (5-15\%) & 20 & 35 & \\
\hline MR moderate (16-25\%) & 19 & 20 & \\
\hline $\begin{array}{l}\text { MR moderate-severe } \\
(26-48 \%)\end{array}$ & 38 & 16 & $<0.001$ \\
\hline \multicolumn{4}{|l|}{$\begin{array}{l}\text { Classifications of LGE, } \mathrm{n} \\
(\%)\end{array}$} \\
\hline None & $24(28)$ & $28(33)$ & \\
\hline Infarct pattern & $19(22)$ & $23(27)$ & \\
\hline Non-infarct pattern & $33(39)$ & $24(28)$ & 0.23 \\
\hline Mixed & $4(5)$ & $3(4)$ & \\
\hline Not done & $5(6)$ & $7(8)$ & \\
\hline \multicolumn{4}{|l|}{ Presence of LGE, n (\%) } \\
\hline LGE present & $56(66)$ & $50(59)$ & \\
\hline LGE absent & $24(28)$ & $28(33)$ & \\
\hline LGE not done & $5(6)$ & $7(8)$ & \\
\hline
\end{tabular}

Data as mean $\pm \mathrm{SD}, \mathrm{n}(\%)$

$L A$ left atrial, $L G E$ late gadolinium enhancement, $L V E D V$ left ventricular end-diastolic volume, $L V E F$ left ventricular ejection fraction, $L V E S V$ left ventricular end-systolic volume, $L V S V$ left ventricular stroke volume, $M R$ mitral regurgitation, $R V E F$ right ventricular ejection fraction, $R V S V$ right ventricular stroke volume

compared with the 'non-improvers'; (6) Baseline MR severity was not associated with long-term mortality.

Our findings are consistent with other large echocardiographic registries such as the Canadian Edwards SAPIEN registry [5], Italian CoreValve registry [14], and PARTNER Trial Cohort A study [15] demonstrating that TAVR is associated with a significant amelioration in MR severity. Although some studies suggested that significant MR results in an increase in mortality rates after TAVR [5, 14, $16,17]$, the findings in our study are consistent with others $[4,7,15,18]$ which have not confirmed this association. Patients with a greater LV mass at baseline and higher aortic valve velocities (i.e. pressure-loaded ventricles) had a higher degree of MR in our study, likely due to raised LVEDP. We postulate that TAVR leads to the reduction of LVEDP and subsequently results in the amelioration of MR.

Interestingly, we found that improvement in MR was neither associated with more favourable cardiac reverse remodeling nor lower mortality rates compared with the 'non-improvers'. There is however the possibility that a 6-month follow-up scan may have been too early to identify any difference in reverse remodeling between the groups. We also found that the presence of LGE at baseline was associated with improvement in MR 6-months post-TAVR. A possible explanation is that patients with significant MR tend to have a more critical AS and a higher trans-valvular gradient, which inevitably results in a higher LV mass and myocardial replacement fibrosis, depicted as LGE. The greater alleviation of ventricular afterload in these patients following TAVR could result in greater LV mass regression and systolic atrioventricular gradient, leading to a greater degree of MR reduction.

A key strength of our study was the use of CMR to reliably quantitate MR volume with low intra- and inter-observer variabilities, irrespective of MR jet geometry [9, 19]. Previous TAVR studies have frequently used transthoracic echocardiography for MR assessment, which has limited reproducibility and relies on mathematical assumptions of LV geometry and cavity size, which may not apply in the remodeled ventricle. In fact, echocardiography, when compared to CMR, was found to overestimate MR severity in many patients [20]. Some studies have also suggested that CMR is more accurate than echocardiography in assessing the severity of MR, especially in those with prolapsing leaflets and eccentric jets [21]. Echocardiographic evaluation of MR severity requires integration of various qualitative and quantitative measurements [22]. The variety of methods used for the quantitative assessment of MR may further explain the discrepancies amongst previous studies [5, 15-18].

The presence of myocardial fibrosis has been reported to be an adverse prognostic marker in patients with AS, with a 6-8 fold increased mortality risk [23, 24]. Myocardial fibrosis has also been shown to adversely affect prognosis and functional outcomes following surgical aortic valve replacement [13], but as yet its role is not fully elucidated in a TAVR population. In a small study $(n=20)$, the presence of LGE was found to predict higher cardiovascular mortality in patients with severe AS undergoing trans-femoral TAVR [25]. The clinical impact of LGE, however, has never been assessed in the setting of concomitant MR in severe AS. We have shown that the presence of LGE was associated with an improvement in MR in the short term (6 months) following TAVR, although the mechanism for this remains undefined.

Despite excellent procedural success and outcomes following TAVR, issues remain regarding optimal patient selection. Decision-making in patients with significant MR in the context of severe AS is often complex. One option is 
Fig. 1 CMR characteristics at baseline and 6-months for 'significant' and 'nonsignificant' MR groups. LVEDV left ventricular end-diastolic volume, LVESV left ventricular end-systolic volume, $M R$ mitral regurgitation

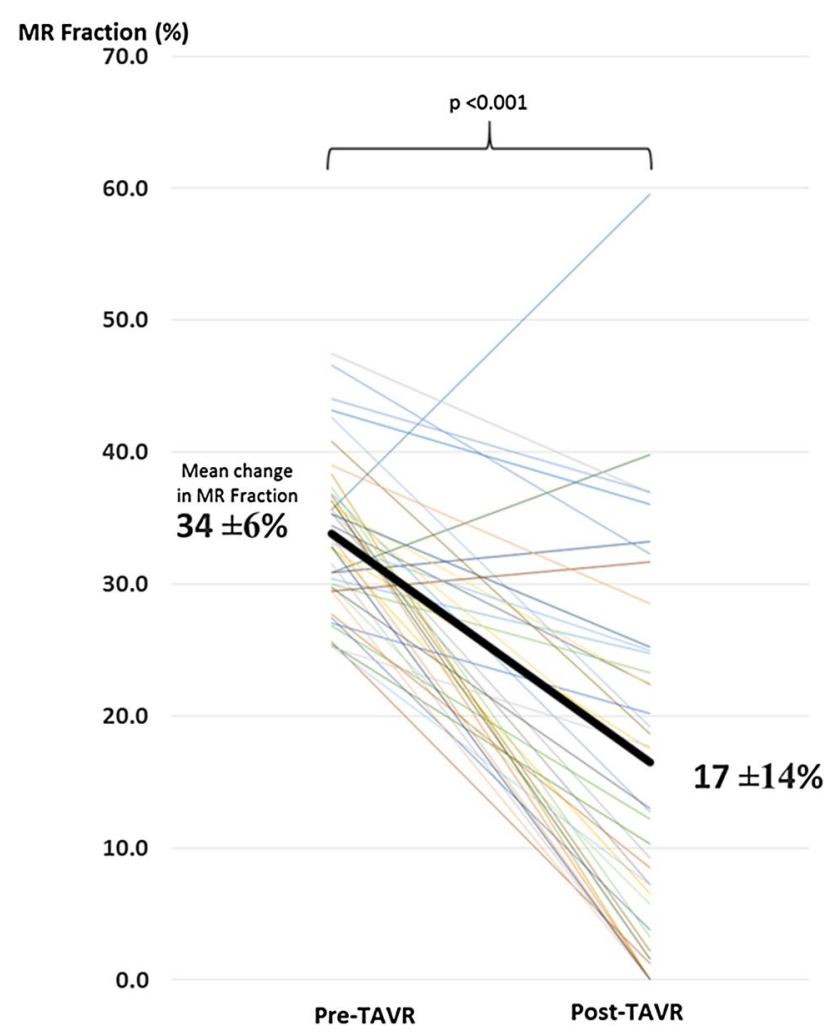

Fig. 2 Change in MR fraction (\%) in the 'significant MR' group post-TAVR. $M R$ mitral regurgitant, TAVR transcatheter aortic valve replacement

to perform a double valve (aortic and mitral) surgical procedure, which might be considered too high-risk in this already high-risk population. The other option is to perform TAVR as a compromise solution, accepting non-treatment of concomitant MR with a potential negative impact on patient outcomes. Therefore, identifying patients with the highest and lowest likelihood for MR improvement could be very important in the clinical decision-making process. LGECMR might allow clinicians to select patients who will most benefit from the TAVR procedure, obviating the need for high-risk double valve surgery. On the other hand, doublevalve surgery may be more appropriate in patients with a low likelihood of MR improvement after TAVR. Although our small sample size did not demonstrate mortality benefits in those who improved their MR status, the literature to date has shown that MR improvement contributes to patient symptomatic improvement [26-28].

\section{Limitations}

The moderate sample size, short follow-up time frame and the single-centre study design limits the strength of our conclusions. However, comparisons between the two groups using the highly reproducible technique of CMR meant it was appropriately powered for LV reverse remodeling parameters. The exclusion of patients with pacemakers (7\%), severe AR and inclusion of survivors only in the CMR analysis raises the potential for selection bias. The analyzed population however did not differ in terms of baseline characteristics from the original whole study population. Because we excluded patients with contraindications to CMR and specific medical conditions, our study population is highly selected and so our conclusions cannot be extrapolated to all patients with severe AS. 
Fig. 3 Change in cardiac reverse remodeling parameters in 'improvers' and 'nonimprovers'. AoFF aortic forward flow, $L V E D V$ left ventricular end-diastolic volume, $L V E F$ left ventricular ejection fraction, $L V E S V$ left ventricular end-systolic volume, $L V S V$ left ventricular stroke volume, $L V S V i$ left ventricular stroke volume indexed, $M R$ mitral regurgitation, Rfraction regurgitant fraction, $R V E S V$ right ventricular end-systolic volume, $R V E D V$ right ventricular enddiastolic volume, $R V E F$ right ventricular ejection fraction, Rvol regurgitant volume, RVSV right ventricular stroke volume

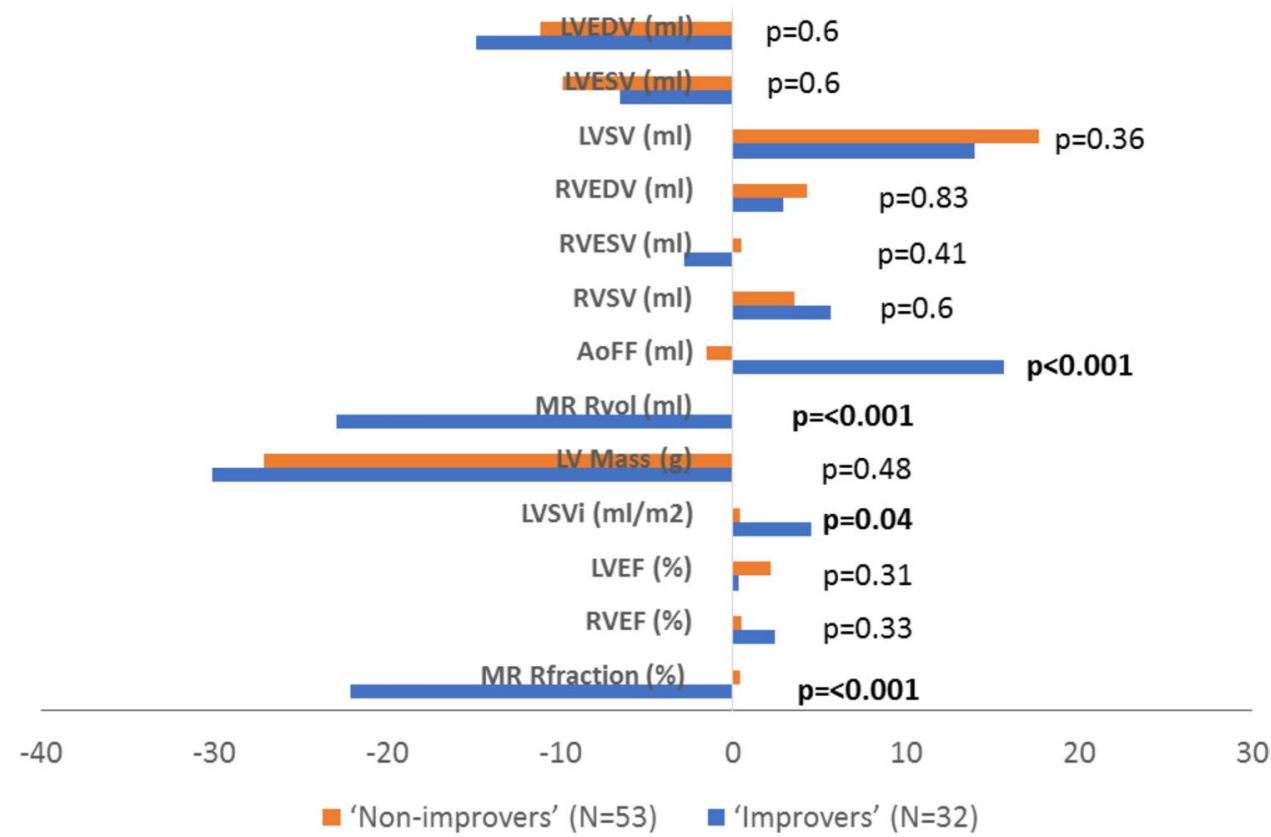

high velocities. When performing phase contrast-based flow measurements in patients with heart valve replacement, there is also a potential for flow and volume miscalculation due to prosthesis-related distortions of the magnetic field [29]. Confounders such as primary or ischemic etiology, change in medications and development of bundle branch block or aortic regurgitation could additionally impact on cardiac reverse remodeling following TAVR. Finally, quantification of fibrosis on LGE images were analyzed using a semi-automatic, signal intensity threshold method rather than the newer T1 mapping techniques, as the latter were not widely employed at the time of patient recruitment.

\section{Conclusion}

\section{Number at risk}

Non-significant MR 43

Significant MR 42

Fig. 4 Kaplan Meier Curve for cumulative survival in 'significant' and 'non-significant MR' groups according to baseline status. Log rank $\mathrm{p}=0.94 . M R$ mitral regurgitation

Additionally, our study had a high proportion of patients with atrial fibrillation (20\%), an arrhythmia which could reduce the quality of image acquisition and therefore reduce the accuracy of volumetric quantification with CMR. MR fraction in the context of severe AS may be overestimated using CMR phase contrast imaging due to underestimation of aortic forward flow when sampling
Significant MR is common in patients undergoing TAVR and improves in the majority post-procedure. Improvement in MR was not associated with LV reverse remodeling and baseline MR severity was not associated with mortality.

Acknowledgements The authors are grateful for the support and assistance of the research nurses (Fiona Richards, Petra Bijsterveld and Lisa Clark) and the radiographers (Gavin Bainbridge, Caroline Richmond and Margaret Saysell) during this project.

Funding This study was part-funded by the British Heart Foundation (PG/11/126/29321) and also the National Institute for Health Research (NIHR) Leeds Clinical Research Facility. The views expressed are those of the author(s) and not necessarily those of the NHS, NIHR or the Department of Health. 


\section{Compliance with ethical standards}

Conflict of interest The authors declare that they have no conflict of interest.

Ethical approval The study was approved by the National Research Ethics Service (08/H1307/106) and complied with the Declaration of Helsinki.

Informed consent All patients provided written informed consent.

Open Access This article is distributed under the terms of the Creative Commons Attribution 4.0 International License (http://creativeco mmons.org/licenses/by/4.0/), which permits unrestricted use, distribution, and reproduction in any medium, provided you give appropriate credit to the original author(s) and the source, provide a link to the Creative Commons license, and indicate if changes were made.

\section{References}

1. Kodali SK, Williams MR, Smith CR et al (2012) Two-year outcomes after transcatheter or surgical aortic-valve replacement. N Engl J Med 366:1686-1695. https://doi.org/10.1056/NEJMo a1200384

2. Fairbairn TA, Meads DM, Mather AN et al (2012) Serial change in health-related quality of life over 1 year after transcatheter aortic valve implantation: predictors of health outcomes. J Am Coll Cardiol 59:1672-1680. https://doi.org/10.1016/j. jacc.2012.01.035

3. Uddin A, Fairbairn TA, Djoukhader IK et al (2015) Consequence of cerebral embolism after transcatheter aortic valve implantation compared with contemporary surgical aortic valve replacement: effect on health-related quality of life. Circ Cardiovasc Interv. https://doi.org/10.1161/CIRCINTERVENTIO NS.114.001913

4. Hutter A, Bleiziffer S, Richter V et al (2012) Transcatheter aortic valve implantation in patients with concomitant mitral and tricuspid regurgitation. Ann Thorac Surg 95:77-84. https://doi. org/10.1016/j.athoracsur.2012.08.030

5. Toggweiler S, Boone RH, Rodés-Cabau J et al (2012) Transcatheter aortic valve replacement: outcomes of patients with moderate or severe mitral regurgitation. J Am Coll Cardiol 59:2068-2074. https://doi.org/10.1016/j.jacc.2012.02.020

6. Sannino A, Losi MA, Schiattarella GG et al (2015) Meta-analysis of mortality outcomes and mitral regurgitation evolution in 4839 patients having transcatheter aortic valve implantation for severe aortic stenosis. Am J Cardiol 114:875-882. https:// doi.org/10.1016/j.amjcard.2014.06.022

7. Wilbring M, Tugtekin SM, Ritzmann M et al (2014) Transcatheter aortic valve implantation reduces grade of concomitant mitral and tricuspid valve regurgitation and pulmonary hypertension. Eur J Cardiothorac Surg 46:818-824. https://doi. org/10.1093/ejcts/ezu037

8. Krieger EV, Lee J, Branch KR et al (2016) Quantitation of mitral regurgitation with cardiac magnetic resonance imaging: a systematic review. Heart 102:1-1870. https://doi.org/10.1136/ heartjnl-2015-309054

9. Aplin M, Kyhl K, Bjerre J et al (2016) Cardiac remodelling and function with primary mitral valve insufficiency studied by magnetic resonance imaging. Eur Heart J 17:jev321. https://doi. org/10.1093/ehjci/jev321
10. Gelfand EVMW (2007) Assessment of valvular heart disease with cardiovascular magnetic resonance. Indian J Radiol Imaging 17:120-132

11. Myerson SG, D’Arcy J, Christiansen JP et al (2016) Determination of clinical outcome in mitral regurgitation with cardiovascular magnetic resonance quantification. Circulation 133:22872296. https://doi.org/10.1161/CIRCULATIONAHA.115.01788 8

12. Kwong RY, Farzaneh-Far A (2011) Measuring myocardial scar by CMR. JACC Cardiovasc Imaging 4:157-160. https://doi. org/10.1016/j.jcmg.2010.12.004

13. Fairbairn TA, Steadman CD, Mather AN et al (2013) Assessment of valve haemodynamics, reverse ventricular remodelling and myocardial fibrosis following transcatheter aortic valve implantation compared to surgical aortic valve replacement: a cardiovascular magnetic resonance study. Heart 99:1185-1191. https://doi.org/10.1136/heartjnl-2013-303927

14. Bedogni F, Latib A, De Marco F et al (2013) Interplay between mitral regurgitation and transcatheter aortic valve replacement with the corevalve revalving system: a multicenter registry. Circulation 128:2145-2153. https://doi.org/10.1161/CIRCULATIO NAHA.113.001822

15. Barbanti M, Webb JG, Hahn RT et al (2013) Impact of preoperative moderate/severe mitral regurgitation on 2-year outcome after transcatheter and surgical aortic valve replacement insight from the placement of aortic transcatheter valve (PARTNER) trial cohort A. Circulation 128:2776-2784. https://doi. org/10.1161/CIRCULATIONAHA.113.003885

16. Haensig M, Holzhey DM, Borger MA et al (2014) Improved mitral valve performance after transapical aortic valve implantation. Ann Thorac Surg 97:1247-1253. https://doi.org/10.1016/j. athoracsur.2013.11.025

17. Khawaja MZ, Williams R, Hung $J$ et al (2014) Impact of preprocedural mitral regurgitation upon mortality after transcatheter aortic valve implantation (TAVI) for severe aortic stenosis. Heart 100:1799-1803. https://doi.org/10.1136/heartjnl-2014305775

18. D'Onofrio A, Gasparetto V, Napodano M et al (2012) Impact of preoperative mitral valve regurgitation on outcomes after transcatheter aortic valve implantation. Eur J Cardiothorac Surg 41:1271-1276. https://doi.org/10.1093/ejcts/ezr236

19. Cawley PJ, Hamilton-Craig C, Owens DS et al (2013) Prospective comparison of valve regurgitation quantitation by cardiac magnetic resonance imaging and transthoracic echocardiography. Circ Cardiovasc Imaging 6:48-57. https://doi.org/10.1161/ CIRCIMAGING.112.975623

20. Gelfand E, Hughes S, Yeon S (2006) Severity of mitral and aortic regurgitation as assessed by cardiovascular magnetic resonance: optimizing correlation with Doppler echocardiography. J Cardiovasc Magn Reson 8:503-507

21. Uretsky S, Gillam L, Lang R et al (2015) Discordance between echocardiography and MRI in the assessment of mitral regurgitation severity: a prospective multicenter trial. J Am Coll Cardiol 65:1078-1088. https://doi.org/10.1016/j.jacc.2014.12.047

22. Chew PG, Bounford K, Plein S et al (2018) Multimodality imaging for the quantitative assessment of mitral regurgitation. Quant Imaging Med Surg 8:342-359. https://doi.org/10.21037 /qims.2018.04.01

23. Dweck MR, Joshi S, Murigu T et al (2011) Midwall fibrosis is an independent predictor of mortality in patients with aortic stenosis. J Am Coll Cardiol 58:1271-1279. https://doi. org/10.1016/j.jacc.2011.03.064

24. Nigri M, Azevedo CF, Rochitte CE et al (2009) Contrastenhanced magnetic resonance imaging identifies focal regions of intramyocardial fibrosis in patients with severe aortic valve 
disease: correlation with quantitative histopathology. Am Heart J 157:361-368. https://doi.org/10.1016/j.ahj.2008.09.012

25. Barone-Rochette G, Piérard S, De Ravenstein CD et al (2014) Prognostic significance of LGE by CMR in aortic stenosis patients undergoing valve replacement. J Am Coll Cardiol 64:144-154. https://doi.org/10.1016/j.jacc.2014.02.612

26. Bach DS, Bolling SF (1995) Early improvement in congestive heart failure after correction of secondary mitral regurgitation in end-stage cardiomyopathy. Am Heart J 129:1165-1170. https ://doi.org/10.1016/0002-8703(95)90399-2

27. D'Ascenzo F, Moretti C, Marra WG et al (2015) Meta-analysis of the usefulness of mitraclip in patients with functional mitral regurgitation. Am J Cardiol 116:325-331. https://doi. org/10.1016/j.amjcard.2015.04.025

28. Maisano F, Franzen O, Baldus S et al (2013) Percutaneous mitral valve interventions in the real world: early and 1-year results from the ACCESS-EU, A prospective, multicenter, nonrandomized post-approval study of the Mitraclip therapy in Europe. J Am Coll Cardiol 62:1052-1061. https://doi. org/10.1016/j.jacc.2013.02.094

29. Richau J, Dieringer MA, Traber J et al (2017) Effects of heart valve prostheses on phase contrast flow measurements in cardiovascular magnetic resonance-a phantom study. J Cardiovasc Magn Reson 19:5. https://doi.org/10.1186/s12968-016-0319-1 